\title{
Correlating Coefficient of Friction and Shear Force to Platen Motor Current in Tungsten and Interlayer Dielectric Chemical Mechanical Planarization at Highly Non-Steady-State Conditions
}

\author{
R. Headley ${ }^{1}$, C. Frank ${ }^{1}$, Y. Sampurno ${ }^{1,2}$ and A. Philipossian ${ }^{1,2}$ \\ ${ }^{1}$ Department of Chemical and Environmental Engineering, \\ University of Arizona, Tucson, Arizona, 85721 USA \\ ${ }^{2}$ Araca Inc, Tucson, Arizona, 85718 USA
}

Correlations between shear force and platen motor current (PMC), as well as those between coefficient of friction (COF) and PMC were investigated for various tungsten and interlayer dielectric (ILD) chemical mechanical planarization (CMP) cases where the processes were highly non steady-state. We chose to initially focus on non-steady-state conditions because we believed the relationships among shear force, $\mathrm{COF}$ and platen motor current to be clearer as opposed to steady-state conditions. Shear force, normal force and PMC data were collected from twelve different Stribeck+ curves at an acquisition frequency of $1,000 \mathrm{~Hz}$ and analyzed in order to determine any emerging trends. For the 12 cases, involving 8 pre-polished blanket CVD tungsten and 4 silicon dioxide blanket wafers, it was discovered that PMC closely mirrored shear force as evidenced by a high average correlation coefficient (0.955) and coefficient of determination (0.916) obtained from all runs. For COF vs. PMC, the average correlation coefficient and coefficient of 
determination for all cases were 0.758 and 0.608 , respectively. These average values were dragged down by 5 cases in which the dominant tribological mechanism was found to be "boundary lubrication" where COF changed minimally with pseudo-Sommerfeld number.

\section{Introduction and Motivation}

For the past 4 decades, and with no end in sight, chemical mechanical planarization (CMP) has enabled IC manufacturing in achieving economically-viable global and local planarity across the entire wafer surface. During CMP, a rotating wafer is pressed onto a rotating pad (often made of a polymeric material) while a slurry containing chemicals and abrasive nanoparticles is applied atop the pad which is then transported to the pad-wafer interface through a combination of polisher kinematics and centrifugal forces aided by the pores and grooves of the pad. When done properly, 3-body contact caused by the pad surface asperities, slurry abrasives nanoparticles and the wafer surface lead to the uniform removal of material from the wafer surface. ${ }^{(1-5)}$

Based on the above description, albeit simplified, it is obvious that tribology ought to be intimately linked to our understanding of various contact-related nuances of the process. An impressive body of experimental and numerical work, that is fundamental in nature, has been published by our research group, as well as others, focusing on the tribological aspects of polishing wafers in tungsten ${ }^{(6-10)}$ and interlayer dielectric (ILD) ${ }^{(11-15)}$ applications. These studies have all focused on the real-time measurement of the pad-slurry-wafer coefficient of friction (COF) through precise extremely reliable, accurate and precise measurement of the shear and normal forces encountered in CMP which required fitting the CMP polisher with costly friction plates (which are quite large 
and complex, and may not always be suitable for certain types of polishers), force sensors, multiplexers and signal amplifiers. The scientific literature is filled with alternate methods ${ }^{(16-30)}$ of inferring the coefficient of friction in CMP with the most popular of such alternate methods centered around measuring platen motor current $(\mathrm{PMC}){ }^{(31-55)}$. During CMP, the wafer and the platen co-rotate at desired velocity setpoints. The pad-slurry-wafer interface undergoes a myriad of 3-body interactions, which due to the frictional forces involved, may facilitate, or resist, platen and wafer rotation. As such, in order for the polisher to control the angular velocities to the desired setpoints, torque motor characteristics must change due to an increase or decrease in the frictional energy loss component. Hence, for rotary-type polishers, the torque current of the carrier and the platen may be monitored using an induction-type loop current sensor attached directly to the DC motor current line, which gives an indication of the frictional forces present in the pad-slurry-wafer interface. While this method is much more cost-effective compared to the traditional COF method noted above, signal-to-noise ratios get somewhat deteriorated due to the many moving parts and gears that at times tend to interfere with the true motor current being captured and recorded. If our research could indicate that PMC data was able to replace shear force data, it would have has widespread positive implications for IC manufacturers because it would enable one to monitor that pad-slurry-wafer interfacial conditions without the need to retrofit polishers used in high-volume manufacturing with expensive ancillary equipment such as friction plates, directional force sensors, multiplexers and signal amplifiers.

During the past 7 years, our research group has performed several tribological studies during which we have generated Stribeck+ curves ${ }^{(56,57)}$ using a single 300 -mm blanket wafer (i.e. deposited with CVD tungsten, ECP copper or PETEOS-based silicon dioxide films) in an effort to shed additional fundamental light on the contact mechanism(s) in CMP. Results of most of these studies have been 
published previously ${ }^{(56,57)}$ where, in parallel with collecting shear and normal force data at 1,000 $\mathrm{Hz}$, we happen to have also collected platen motor current (PMC) data at the same frequency. It must be noted that we have never analyzed, nor reported on, this metric. In our present study, for each of these studies involving Stribeck + curve generation, we set out to systematically: (a) Define details of the respective process conditions; (b) Analyze all shear force and PMC data-pairs along with all the COF and PMC data-pairs as a function of polish time; (c) Correlate the experimental outputs to each other; and (d) Draw conclusions from the results.

\section{Experimental Apparatus}

An Araca APD-800 polisher and tribometer was used to perform all tungsten and copper polishing experiments. ${ }^{(58)}$ This system is a single-platen research and development polisher and tribometer designed to process substrates that are 200 as well as $300 \mathrm{~mm}$ in diameter. The APD-800 is equipped with signal acquisition hardware such as direction-dependent load cells, as well as Araca Inc.'s FSX-800 proprietary signal analysis software. This feature provides the unique ability to acquire accurate real-time shear force and down force measurements (in our experiments, data capture frequency for all parameters was set at $1,000 \mathrm{~Hz}$, although we could have used frequencies as high as $2,300 \mathrm{~Hz}$ at the expense of requiring a much larger computational power). Additionally, the APD-800 contains a single-point infrared (IR) temperature sensor used for measuring real-time pad surface temperature data during polishing. On this tool, the non-oscillating counterclockwise wafer carrier and its drive mechanism sit on a friction table attached to the frame. A load cell connected to the table measures the main component $\mathrm{F}_{\mathrm{y}}$ of the friction force vector. This component runs perpendicular to the line connecting the platen and carrier centers. The radial 
component $F_{x}$ of the friction force is not measured but is known to be small from experiments on a similar $200 \mathrm{~mm}$ tool. ${ }^{(59)}$ The head does not use a contacting retaining ring (which is why our CMP processes run at somewhat lower temperatures compared to those in HVM). Instead, a noncontact wafer backing film attached to a water-filled template holds the wafer. The platen and its drive mechanism sit on four load cells that monitor the total force $\mathrm{F}_{\mathrm{z}}$ transmitted by the wafer to the pad. This includes both the applied load and any fluid forces that develop in the interface. All forces are measured at $1,000 \mathrm{~Hz}$. There is no feedback between the normal force load cells and the carrier. Inside of the carrier, behind the wafer, there is an air chamber that applies the polishing force. An electro-pneumatic transducing regulator controls the chamber pressure. Pressure adjustments can occur but are much slower than the force acquisition frequency. The applied load is periodically checked and calibrated on a static platen. A more detailed description of the polishing system can be found elsewhere. ${ }^{(58)}$ In addition to the force acquisition hardware and software, the APD-800 is also equipped with carrier, platen and conditioner motor currents data acquisition, amplification and recording hardware all capable of data acquisition at up to 2,300 $\mathrm{Hz}$. In all of our tests, we measured these metrics at $1,000 \mathrm{~Hz}$ and have chosen to only report on the platen motor current (PMC) due to its higher signal-to-noise ratio compared to the other two motor currents. It must be noted that the acquisition clocks that managed the shear force and PMC data were off by appx. $250 \mathrm{~ms}$ from each other. We had to take this into account during our signal analysis and reporting. 


\section{Experimental and Analytical Procedures}

In this report, 12 different and highly non-steady cases (denoted as Cases 1 - 12) were studied. The non-steady state conditions were determined by systematically varying the sliding pressure and velocities in a given process throughout predetermined polish times. The blanket wafer type, pad, slurry, conditioning pad, conditioning process recipe, and polishing time were varied depending on the specific case.

For Cases $1-6,300-\mathrm{mm}$ pre-polished blanket CVD tungsten wafers were used together with DowDupont IC1000 K Groove pads. For Cases 7 and 8, the pre-polished blanket CVD tungsten wafers were $200 \mathrm{~mm}$ in diameter also in conjunction with DowDupont IC1000 K groove pads. For Cases 9 - 12, we used 300-mm plasma enhanced tetraethoxyorthosilicate (PETEOS) based blanket silicon dioxide wafers.

For the first six cases, the hydrogen peroxide based slurries used were Versum DP1142-1 (Case 1), Versum DP1142-2 (Case 2), Versum W5880-1 (Case 3), Versum W5880-2 (Case 4), Versum W5880-3 (Case 5), and CMC W7801 (Case 6) all flowing at $250 \mathrm{cc}$ per minute. In these cases, pads were broken-in for 30 minutes using a Saesol 4DNS80AMC1 disc rotating at 70 RPM with a generic sinusoidal sweep schedule. The rotational velocity of the pads was maintained at 80 RPM. In between polishes, ex-situ conditioning was performed for 30 seconds at a downforce of $6.75 \mathrm{lb}_{\text {f. }}$ The DP1142-1 and DP1142-2 slurries were high-selectivity (to silicon nitride) slurries with varying amounts of a certain proprietary additives. As for W5880-1, W5880-2 and W5880-3 slurries, they had varying amounts of colloidal silica nano-particles $(1.5,2.5$ and 3.5 percent by weight, respectively) and were especially formulated so that there were no detectable changes in their formulation chemistries. 
For Cases 7 and 8 , slurry flow rate was $200 \mathrm{cc}$ per minute. Here, the slurry used was also the CMC W7801. For Case 7, the pad was conditioned by using a 3M A165 CIP disc, and for Case 8, the conditioner was an MGAM 4S845P4F5 disc. Both discs rotated at 95 RPM and the down force for each disc was $10 \mathrm{lb}_{\mathrm{f}}$ with a sinusoidal sweep schedule in which the disc swept across the pad 10 times per min.

Case 9 used a CMC D100 concentrically grooved pad with a Merck Klebosol 1508-30 slurry without any dilution (i.e. at $30 \%$ by weight solids) at a flow rate of $300 \mathrm{cc}$ per min. The pad was conditioned using a 3M A165 disc rotating at 95 RPM and sweeping using a sinusoidal schedule in which the disc swept across the pad 10 times per min. In-situ conditioning was performed with a down force of $26.7 \mathrm{~N}$.

For Cases $10-12$, different pads were used. Case 10 used a CMC D100 concentrically grooved pad, Case 11 used a DowDupont IC1000 K Grooved pad, and Case 12, a DowDuPont IC1020 M Grooved pad. These 3 cases used a Fujimi PL-4217 diluted slurry with ultra-pure water (UPW) to a final solids concentration of $10 \%$ by weight with a slurry flow rate of $300 \mathrm{cc}$ per minute. The pads were conditioned using a 3M A165 disc rotating at 95 RPM and sweeping using a sinusoidal schedule with a sweep frequency of 10 times per minute. The in-situ pad conditioning was conducted with a downforce of $26.7 \mathrm{~N}$.

The pressures (in PSI) and sliding velocities (in $\mathrm{m} / \mathrm{s}$ ) for each of the twelve cases are listed in Tables I (Case 1 - 3), II (Cases 4 - 6), III (Cases 7 - 8), IV (Case 9), and V (Cases 10 - 12). In this study, normal force, shear force, and PMC data were collected at 1,000 Hz.

As mentioned in the "Introduction and Motivation" section of this paper, the PMC data had never been reported, nor analyzed, until now. By using the computing power of MATLAB R 2018b, a 
precise and accurate examination of the trends between the shear force, COF and PMC data was conducted. Accurate and efficient examination of the emerging trends from millions of shear force, normal force and PMC data points was made possible with MATLAB R 2018b. After importing the raw data into MATLAB, we extracted real-time measurements of platen motor current (PMC) and shear force, as well as PMC and COF (which is the ratio of shear to normal force), from the acquisition program. Before conducting any statistical analyses, raw PMC data was shifted by 250 ms to account for the known difference between the two data acquisition clocks as noted earlier. Also, in all cases, velocity and pressure ramp-up and ramp-down sections occurring in the first and last five seconds of the entire process were excluded due to the overwhelming presence of noise in those regions.

As mentioned earlier, in this study we wanted to not only correlate COF to platen motor current, but shear force to PMC as well. The former carries a greater mechanistic significance as it is a key parameter that appears in almost all material removal rate models either by itself or lumped into a mechanical proportionality constant. ${ }^{(60,61)}$ However, since COF contains information about normal force and associated vertical vibrations, data interpretation can at times be complicated. On the other hand, correlating shear force to PMC would allow for analyzing data based on the horizontal torque applied to the platen (as measured by PMC) as a function of shear force in the pad-slurry-wafer interface. 


\section{Results and Discussion}

Figs. 1 - 12 summarize the results obtained from all cases studied. On the top left-hand-side of each figure, shear force, normal force and PMC values are plotted vs. time. In each case, the first and last five seconds of each run have been excluded from the correlation plots, that is, only the data bound between the two dashed lines was used to generate the correlation plots shown on the right. We had found out earlier that the initial 5-sec ramp-up and final 5-sec ramp-down regions skewed the data because of the excessive amounts of normal force and normal vibrations. On the bottom left-hand-side of each figure, we show PMC values vs. time. On the top right-hand-side of each figure is the PMC vs. shear force correlation plots which indicate the correlation coefficient (R), the coefficient of determination $\left(\mathrm{R}^{2}\right)$, and the respective regression line. On the bottom righthand-side of each figure is the PMC vs. COF correlation plots which indicate the correlation coefficient $(R)$, the coefficient of determination $\left(R^{2}\right)$, and the respective regression line. The data values for both correlation plots are the values in between the dashed line shown on the two plots to the left on each figure.

Regarding shear force vs. PMC, the average correlation coefficient and coefficient of determination for all 12 studied cases were 0.955 and 0.916 , respectively. This indicated that, for the predetermined non-steady-state processes being investigated, shear force data could be replaced with PMC data with a high degree of confidence regardless of the type of processes, wafers, and consumables sets used. However, for COF vs. PMC, the average correlation coefficient and coefficient of determination for all 12 cases studied were much lower at 0.758 and 0.608, respectively. The lowest coefficients were observed for Cases $6-10$ (ranging from 0.355 to 0.714 for $\mathrm{R}$, and from 0.126 to 0.509 for $\mathrm{R}^{2}$ ) indicating that when we accounted for normal forces (by calculating COF), the correlations worsened. The reason behind the strong correlations 
seen in Cases $1-5,11$ and 12, and the lackluster correlations seen in Cases $6-10$ are best explained by a closer examination of the Stribeck+ curves themselves as shown in Fig. $13-15$. For Cases $1-5,11$ and 12, because each process is in "mixed lubrication", or in "boundary lubrication" that quickly transitions to "mixed lubrication" (62-64), instantaneous COF values change greatly with the pseudo-Sommerfeld number thus allowing a strong correlation between COF and PMC. On the other hand, for Cases $6-10$, Stribeck + curves are mostly, if not entirely, in "boundary lubrication" where COF changes only slightly with pseudo-Sommerfeld number. The reasons as to why certain sets of consumables cause the tribological mechanism to be "boundary lubrication" or one that transitions to "mixed lubrication" have been explained in detail in our previous publications. ${ }^{(1,4,44)}$ In Cases $6-10$, even though we know that shear forces change dramatically as a function of pseudo-Sommerfeld number, their normal force counterparts change proportionately as well. This fact causes minimal changes in COF as a whole and grossly deteriorates the correlation coefficient and the coefficient of determination values.

\section{Conclusion}

This study investigated the correlation between shear force and PMC, as well as that between COF and PMC during highly non-steady-state conditions in tungsten and interlayer dielectric (ILD) CMP processes. The non-steady conditions were created by rapidly changing pressures and velocities within a given run. For the 12 cases studied ( 8 for tungsten and 4 for ILD), we found that real-time PMC data closely mimicked shear force data as evident from high average values of correlation coefficient and coefficient of determination for all 12 cases. This dependence was intuitive as increasing the load increased the shear force which in turn required motor current to increase in order to keep the rotational velocity of the platen constant. The converse was also true 
as decreasing the load caused motor current to drop. As for COF vs. PMC, average correlation coefficient and coefficient of determination for 7 of the 12 cases was quite high. However, for the remaining 5 cases, correlation coefficients degraded significantly. The reason behind the strong correlations seen in the 7 cases, and the lackluster correlation seen in the other 5 cases were explained based on the particulars of the dominant tribological mechanism for each case. In summary, for the non-steady-state processes studied, it became clear that shear force data could be replaced with PMC data with a high degree of confidence regardless of the type of processes, wafers, and consumable sets used. Adopting PMC data in this way has widespread implications for IC manufacturers, most notably for monitoring the conditions at the pad-slurry-wafer interface without the need for installing costly friction plates (that are quite large and complex and may not always be suitable for certain types of polishers), force sensors, multiplexers and signal amplifiers.

\section{References}

1. C. Stuffle, L. Vázquez Bengochea, Y. Sampurno, A. Philipossian (2019). Ultra-Rapid Determination of Material Removal Rates Based Solely on Tribological Data in Chemical Mechanical Planarization. ECS Journal of Solid State Science and Technology. 8. P3035P3039. 10.1149/2.0061905jss.

2. R. Han, Y. Sampurno, M. Bahr, L. Borucki, and A. Philipossian. Effect of Conditioning and Slurry Application Method on Silicon Dioxide Removal Rates Using a Ceria-Based Chemical Mechanical Planarization Slurry. ECS Journal of Solid State Science and Technology 2017 6: P477-P482. 
3. Y. Sampurno, A. Rice, Y. Zhuang, A. Philipossian (2011). An Approach for Correlating Friction Force and Removal Rate to Pad Topography during Tungsten Chemical Mechanical Planarization. Electrochemical and Solid-State Letters. 14. H318. $10.1149 / 1.3589984$.

4. Philipossian, S. Olsen (2003). Fundamental Tribological and Removal Rate Studies of ILD Chemical Mechanical Planarization. Japanese Journal of Applied Physics. 42. 6371-6379. 10.1143/JJAP.42.6371.

5. Bhushan (2013). Introduction to Tribology. John Wiley and Sons.

6. R. Han, Y. Mu, Y. Sampurno, Y. Zhuang, A. Philipossian (2017). Effect of Pad Surface Micro-Texture on Tribological, Thermal and Kinetic Characterizations during Copper Chemical Mechanical Planarization. ECS Journal of Solid State Science and Technology. 6. P201-P206. 10.1149/2.0021705jss.

7. Y. Jiao, X. Liao, C. Wu, Y. Zhuang, Y. Sampurno, A. Philipossian, S. Theng, M. Goldstein (2012). Tribological, Thermal and Kinetic Attributes of 300 vs. $450 \mathrm{~mm}$ Chemical Mechanical Planarization Processes. Journal of the Electrochemical Society. 159. 272-277. 10.1109/ASMC.2012.6212910.

8. D. DeNardis, J. Sorooshian, M. Habiro, C. Rogers, A. Philipossian (2003). Tribology and Removal Rate Characteristics of Abrasive-Free Slurries for Copper CMP Applications. Japanese Journal of Applied Physics. 42. 6809-6814. 10.1143/JJAP.42.6809.

9. A. Philipossian, L. Mustapha (2004). Tribological Attributes of Post-CMP Brush Scrubbing. Journal of The Electrochemical Society - J ELECTROCHEM SOC. 151. $10.1149 / 1.1753586$. 
10. Z. Li, P. Lefevre, I. Koshiyama, K. Ina, D. Boning, A. Philipossian (2005). Comparison of Copper Disc and Copper Wafer Polishing Processes in Terms of Their Kinetic, Tribological, and Thermal Characteristics. Semiconductor Manufacturing, IEEE Transactions on. 18. 681 - 687. 10.1109/TSM.2005.858522.

11. A. Philipossian, S. Olsen (2004). Effect of Slurry Flow Rate on Pad Life during Interlayer Dielectric CMP. Journal of The Electrochemical Society - J ELECTROCHEM SOC. 151. $10.1149 / 1.1723501$.

12. X. Wei, Y. Zhuang, Y. Sampurno, F. Sudargho, C. Wargo, L. Borucki, A. Philipossian (2010). Tribological, Thermal, and Wear Characteristics of Poly(phenylene sulfide) and Polyetheretherketone Retaining Rings in Interlayer Dielectric CMP. Electrochemical and Solid-State Letters. 13. H391. 10.1149/1.3483752.

13. Y. Sampurno, X. Gu, T. Nemoto, Y. Zhuang, A. Teramoto, A. Philipossian, T. Ohmi (2010). End-Point Detection of Ta/TaN Chemical Mechanical Planarization via Forces Analysis. Japanese Journal of Applied Physics. 49. 05FC01. 10.1143/jjap.49.05fc01.

14. C. Stuffle, R. Han, Y. Sampurno, S. Theng, Wei-Tsu Tseng, and A. Philipossian Application of a Slurry Injection System to Cobalt "Buff Step" Chemical Mechanical Planarization. ECS Journal of Solid State Science and Technology 2018 7: P170-P174.

15. H. Jacobsen, E. Stachowiak, G. Zwicker, W. Lortz, R. Brandes (2011). Metrological Assessment of the Coefficient of Friction of Various Types of Silica Using the Motor Current During ILD-CMP. MRS Proceedings. 816. 10.1557/PROC-816-K3.3.

16. L. Borucki, A. Philipossian, Y. Zhuang (2007). Physics of the Coefficient of Friction in CMP. Transactions on Electrical and Electronic Materials. 8. 79-83. 10.4313/TEEM.2007.8.2.079. 
17. T. Fujita, K. Kitade, T. Yokoyama. (2011). Development of Original End Point Detection System Utilizing Eddy Current Variation Due to Skin Effect in Chemical Mechanical Polishing. Japanese Journal of Applied Physics. 50. 05EC09. 10.7567/JJAP.50.05EC09.

18. T. Fujita, K. Kitade (2017). Verification of Operation Principle on End Point Detection by Skin Effect in Chemical Mechanical Planarization. ECS Journal of Solid State Science and Technology. 6. P535-P541. 10.1149/2.0201708jss.

19. M. Bahr, Y. Sampurno, R. Han, A. Philipossian (2017). Improvements in Stribeck Curves for Copper and Tungsten Chemical Mechanical Planarization on Soft Pads. ECS Journal of Solid State Science and Technology. 6. P290-P295. 10.1149/2.0241705jss.

20. L. Borucki, Z. Li, A. Philipossian (2004). Experimental and Theoretical Investigation of Heating and Convection in Copper Polishing. Journal of The Electrochemical Society. 151. G559-G563. 10.1149/1.1774489.

21. D. Rosales-Yeomans, D. DeNardis, L. Borucki, A. Philipossian (2008). Design and Evaluation of Pad Grooves for Copper CMP. Journal of The Electrochemical Society - J ELECTROCHEM SOC. 155. 10.1149/1.2963268.

22. D. Hetherington, D. J. Stein, J. P. Lauffer, E. E. Wyckoff, D. M. Shingledecker (1999). Analysis of in-situ vibration monitoring for end-point detection of CMP planarization processes. Proceedings of SPIE - The International Society for Optical Engineering. 3743. $10.1117 / 12.346902$.

23. J. Sorooshian, D. Hetherington, A. Philipossian (2004). Effect of Process Temperature on Coefficient of Friction during CMP. Electrochemical and Solid State Letters ELECTROCHEM SOLID STATE LETT. 7. 10.1149/1.1792240. 
24. S.Y. Kim, K. J. Lee, Y. Seo (2003). In-situ end point detection of the STI-CMP process using a high selectivity slurry. Microelectronic Engineering. 66. 463-471. 10.1016/S01679317(02)00929-2.

25. M. Helu, J. Chien, D. Dornfeld (2014). In-situ CMP Endpoint Detection Using Acoustic Emission. Procedia CIRP. 14. 454-459. 10.1016/j.procir.2014.03.025.

26. H. Hocheng, Y. L. Huang (2007). In Situ Endpoint Detection by Acoustic Emissions in Chemical-Mechanical Polishing of Metal Overlay. Semiconductor Manufacturing, IEEE Transactions on. 20.306 - 312. 10.1109/TSM.2007.901406.

27. N. Kimura, F. Sakata, T. Tamami. Polishing endpoint detection method. (1997). US Patent 05639388.

28. H. Lee, D. DeNardis, A. Philipossian, Y. Seike, M. Takaoka, K. Miyachi, S. Furukawa, A. Terada, Y. Zhuang, L. Borucki (2007). Thermal, Tribological, and Removal Rate Characteristics of Pad Conditioning in Copper CMP. Transactions on Electrical and Electronic Materials. 8. 67-72. 10.4313/TEEM.2007.8.2.067.

29. J. Yi, C. Xu. Methods for monitoring and controlling chemical mechanical planarization. (2005).US Patent 06931330.

30. Y. Sampurno, L. Borucki, Y. Zhuang, D. Boning, A. Philipossian (2005). A Method for Direct Measurement of Substrate Temperature During Copper CMP. Journal of The Electrochemical Society. 152. G537-G541. 10.1149/1.1925070.

31. X. Chi, G. Dongming, J. Zhuji, R. Kang (2010). A signal processing method for the friction-based endpoint detection system of a CMP process. Journal of Semiconductors. 31. $126002.10 .1088 / 1674-4926 / 31 / 12 / 126002$. 
32. B. Lin, S. Lee (1999). An effective end point detector on oxide CMP by motor current. 295 - 298. 10.1109/ASMC.1999.798248.

33. S. Kim, C. Park, Y. Seo (2003). Signal analysis of the end point detection method based on motor current. Microelectronic Engineering. 66. 472-479. 10.1016/S01679317(02)00930-9.

34. H. Kai Li, T. Qing Wang, D. Wen Zhao, J. Bin Luo, X. Chun Lu (2017). An In Situ End-Point Detection System Using Motor Power Signal for Chemical Mechanical Planarization Process. Key Engineering Materials. 739. 75-80. 10.4028/www.scientific.net/KEM.739.75.

35. Y. Seo, W. Lee, J. Park, S. Kim (2003). Motor-Current-Based Real-Time End Point Detection of Shallow-Trench-Isolation Chemical Mechanical Polishing Process Using High-Selectivity Slurry. Japanese Journal of Applied Physics. 42. 6396-6399. 10.1143/JJAP.42.6396.

36. P. Beckage, R. Lukner, W. Cho, K. Edwards, M. Jester, S. Shaw (1999). Improved metal CMP endpoint control by monitoring carrier speed controller output or pad temperature. Proceedings of SPIE - The International Society for Optical Engineering. $10.1117 / 12.361299$.

37. J. Sorooshian, A. Philipossian, D. Stein, R. Timon, D. Hetherington (2005). Dependence of Oxide Pattern Density Variation on Motor Current Endpoint Detection during Shallow Trench Isolation Chemical Mechanical Planarization. Japanese Journal of Applied Physics. 44. 10.1143/JJAP.44.1219. 
38. C. Kuo, C. Ho, C. Tsai, S. Shih, C. Wu, \& J. Lin, P. Lee (2006). Effects of pad features on motor current EPD signals and the performance of STI CMP process. 2006 Proceedings 23rd International VLSI Multilevel Interconnection Conference, VMIC 2006. 451-455.

39. C. Xu, D. Ming Guo, R. Kang, Z. Ji Jin, F. Wei Huo (2008). Friction-Based In Situ Endpoint Detection of Copper CMP Process. Advanced Materials Research. 53-54. 125130. 10.4028/www.scientific.net/AMR.53-54.125.

40. Bello, M. Wedlake. Methods and systems for chemical mechanical planarization endpoint detection using an alternating current reference signal. (2015). US Patent 10343253.

41. S. Sandhu, L. D. Schultz, D. Laurence D, T. T. Doan. Apparatus for endpoint detection during mechanical planarization of semiconductor wafers. (1991). US Patent 05069002.

42. D. Stein, D. Hetherington (2001). Recent advances in endpoint and in-line monitoring techniques for chemical-mechanical polishing processes. Proceedings of SPIE - The International Society for Optical Engineering. 4406. 10.1117/12.425261.

43. D. Boning, X. Xie, J. Sorooshian, A. Philipossian, D. Stein, D. Hetherington (2019). Relationship Between Patterned Wafer Topography Evolution and STI CMP Motor Current Endpoint Signals.

44. C. Stuffle, R. Han, Y. Sampurno, D. Slutz, S. Theng, L. Borucki \& A. Philipossian. Effect of CVD-Coated Diamond Discs on Pad Surface Micro-Texture and Polish Performance in Copper CMP. ECS Journal of Solid State Science and Technology, 7 (2) P9-14 (2018).

45. P. W. Carter, J. P. Chamberlain. CMP process involving frequency analysis-based monitoring. (2002).US Patent 06431953

46. R. B. Clark-Phelps. System and method for end-point detection in a multi-head CMP tool using real-time monitoring of motor current. (2001). US Patent 06293845. 
47. T. Tamami, F. Sakata, N. Kimura, M. Kodera, A Shigeta. Method and apparatus for determining endpoint during a polishing process. (1998). US Patent 05830041.

48. G. Dongming, X. Chi, R. Kang, J. Zhuji (2011). Model analysis and experimental investigation of the friction torque during the CMP process. Journal of Semiconductors. 32. 036002 . $10.1088 / 1674-4926 / 32 / 3 / 036002$.

49. H. Lee, Y. Kim, H. Kim, H. Jeong (2008). Effect of Process Parameters on Friction Force and Material Removal in Oxide Chemical Mechanical Polishing. Japanese Journal of Applied Physics. 47. 8771-8778. 10.1143/JJAP.47.8771.

50. S. McClatchie, P. Norton, X. Pham, R. Zhou. Methods of and apparatus for controlling polishing surface characteristics for chemical mechanical polishing. (2006).US Patent 07040954.

51. T. Bibby, K. Holland (1998). Endpoint detection for CMP. Journal of Electronic Materials. 27. 1073-1081. 10.1007/s11664-998-0140-1.

52. Li, Hongkai, Xinchun Lu, and Jianbin Luo. "Motor Power Signal Analysis for End-Point Detection of Chemical Mechanical Planarization." Micromachines 8.6 (2017): 177

53. D. Boning, X. Xie, L. Sorooshian, A. Philipossian, D. Stein, D. Hetherington (2019). Relationship Between Patterned Wafer Topography Evolution and STI CMP Motor Current Endpoint Signals.

54. B. Lin, S. Lee (1999). An effective end point detector on oxide CMP by motor current. 295 - 298. 10.1109/ASMC.1999.798248.

55. D. Stein, D. Hetherington (1999). Prediction of tungsten CMP pad life using blanket removal rate data and endpoint data obtained from process temperature and carrier motor 
current measurements. Proceedings of SPIE - The International Society for Optical Engineering. 3743. 10.1117/12.346904.

56. R. Han, Y. Sampurno, S. Theng, F. Sudargho, Y. Zhuang, and A. Philipossian Application of the Stribeck + Curve in Silicon Dioxide Chemical Mechanical Planarization. ECS Journal of Solid State Science and Technology 2017 6: P161-P164.

57. M. Bahr, Y. Sampurno, R. Han, A. Philipossian (2017). Improvements in Stribeck Curves for Copper and Tungsten Chemical Mechanical Planarization on Soft Pads. ECS Journal of Solid State Science and Technology. 6. P290-P295. 10.1149/2.0241705jss.

58. Araca, Inc. aracainc.com/equipment/r-and-d-polishers/.

59. Y. Sampurno, Ph.D. dissertation, University of Arizona, Tucson, AZ (2008).

60. D. Dowson. (1979). History of tribology. Addison-Wesley Longman Limited.

61. P. J. Blau. (2001). The Significance and Use of the Friction Coefficient. Tribology International. 34.9 (2001): 585-91.

62. S. Joo, H. Lian. (2013). Tribology in Chemical-Mechanical Planarization. In: P. Menezes, M. Nosonovsky, S. Ingole, S. Kailas, M. Lovell. (eds) Tribology for Scientists and Engineers.

63. H. Liang. (2005). Chemical Boundary Lubrication in Chemical-Mechanical Planarization. Tribology International. doi:10.1016/j.triboint.2004.08.006.

64. H. Liang, G. H. Xu. (2002). Lubricating Behavior in Chemical-Mechanical Polishing of Copper. doi:10.1016/s1359-6462(01)01249-0. 
Table I. Polishing Conditions for Cases 1 and 2

\begin{tabular}{ccc} 
CMP Step & Pressure (P, PSI) & Sliding Velocity $(\mathrm{v}, \mathrm{m} / \mathrm{s})$ \\
\hline 1 & 2 & 1.4 \\
2 & 2 & 1.8 \\
3 & 4 & 1.0 \\
4 & 3 & 1.8 \\
5 & 4 & 1.8
\end{tabular}

Table II. Polishing Conditions for Cases 3 - 6

\begin{tabular}{ccc} 
CMP Step & Pressure (P, PSI) & Sliding Velocity $(\mathrm{v}, \mathrm{m} / \mathrm{s})$ \\
\hline 1 & 2 & 1.4 \\
2 & 2 & 1.8 \\
3 & 4 & 1.0 \\
4 & 3 & 1.8 \\
5 & 4 & 1.8
\end{tabular}

Table III. Polishing Conditions for Cases 7 and 8

\begin{tabular}{ccc} 
CMP Step & Pressure (P, PSI) & Sliding Velocity $(\mathrm{v}, \mathrm{m} / \mathrm{s})$ \\
\hline 1 & 1.8 & 1.0 \\
2 & 3.2 & 1.0 \\
3 & 1.8 & 1.8 \\
4 & 1.8 & 2.0 \\
5 & 2.5 & 1.8
\end{tabular}


Table IV. Polishing Conditions for Case 9

\begin{tabular}{ccc} 
CMP Step & Pressure (P, PSI) & Sliding Velocity $(\mathrm{v}, \mathrm{m} / \mathrm{s})$ \\
\hline 1 & 6 & 0.6 \\
2 & 4 & 0.6 \\
3 & 6 & 1.0 \\
4 & 4 & 1.0 \\
5 & 6 & 1.5 \\
6 & 2 & 0.6 \\
7 & 4 & 1.5 \\
8 & 2 & 1.0 \\
9 & 4 & 1.5
\end{tabular}

Table V. Polishing Conditions for Case 10 - 12

\begin{tabular}{ccc} 
CMP Step & Pressure (P, PSI) & Sliding Velocity $(\mathrm{v}, \mathrm{m} / \mathrm{s})$ \\
\hline 1 & 6 & 0.6 \\
2 & 4 & 0.6 \\
3 & 6 & 1.0 \\
4 & 4 & 1.0 \\
5 & 6 & 1.5 \\
6 & 2 & 0.6 \\
7 & 4 & 1.5 \\
8 & 2 & 1.0 \\
9 & 4 & 1.5
\end{tabular}



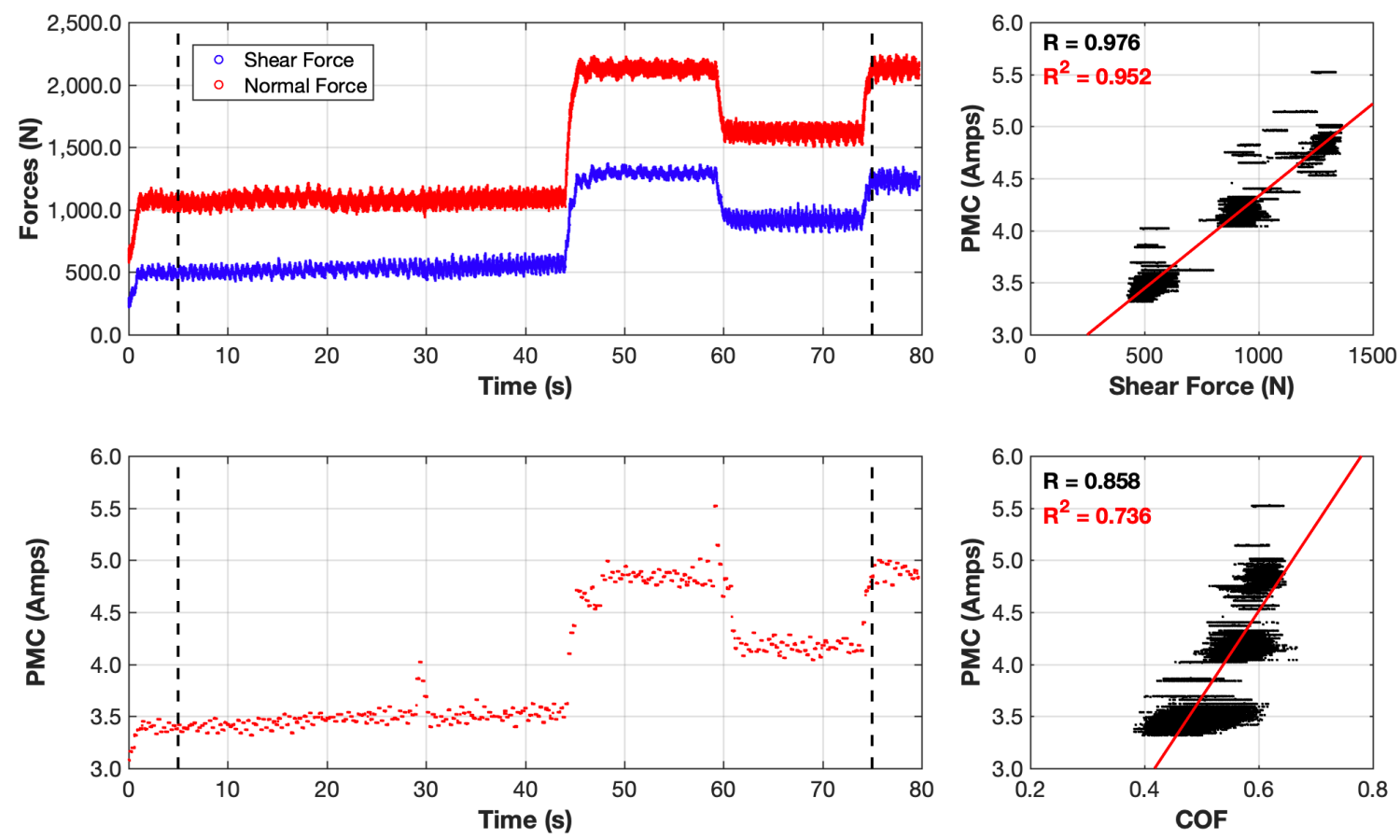

Figure 1. Case 1 - Shear Force and Normal Force vs. Time (top left), Shifted PMC vs. Time (bottom left), Correlation Plot of Shifted PMC Data vs. Shear Force Data (top right), Correlation Plot of Shifted PMC Data vs. COF Data (bottom right).
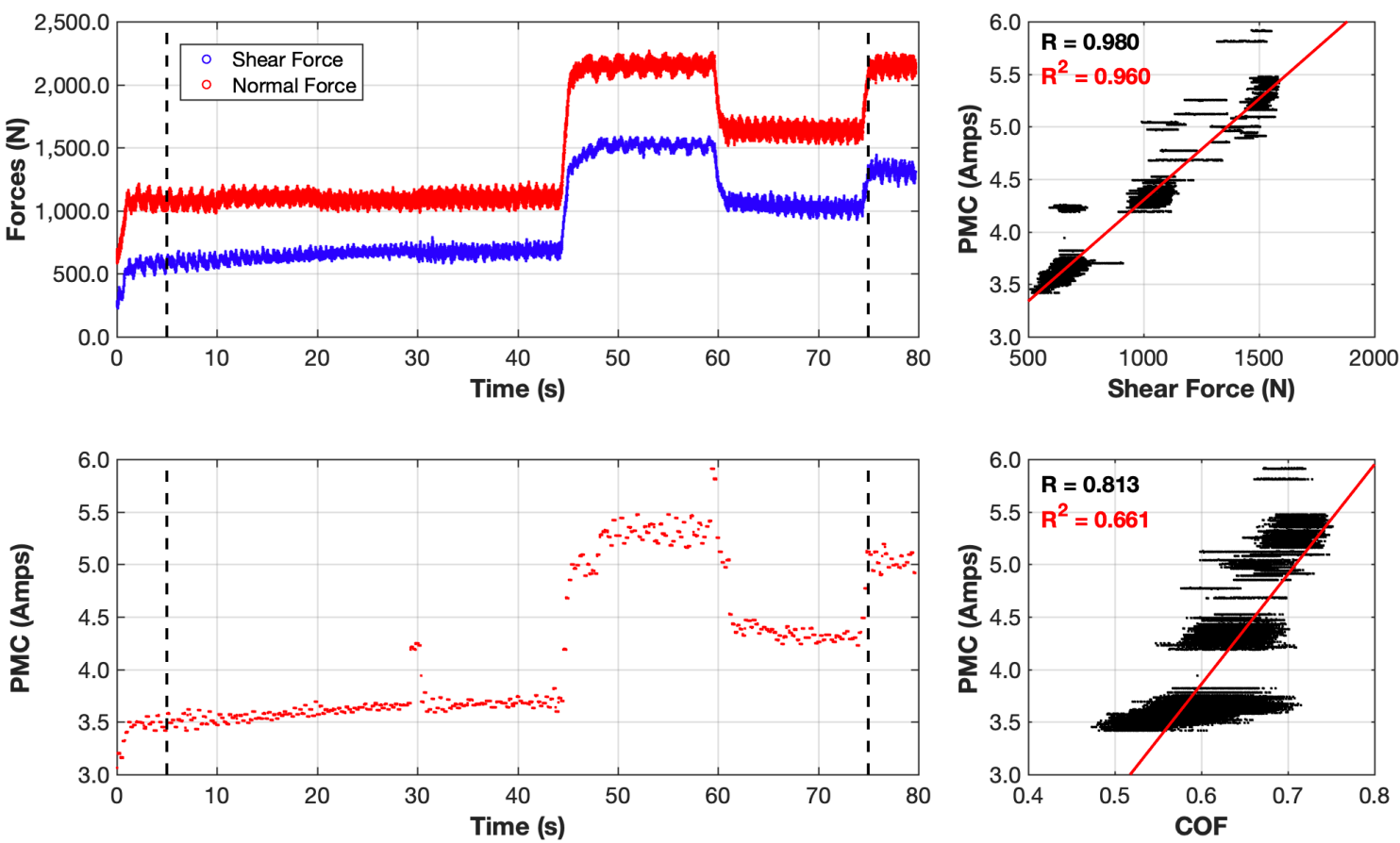

Figure 2. Case 2 - Shear Force and Normal Force vs. Time (top left), Shifted PMC vs. Time (bottom left), Correlation Plot of Shifted PMC Data vs. Shear Force Data (top right), Correlation Plot of Shifted PMC Data vs. COF Data (bottom right). 

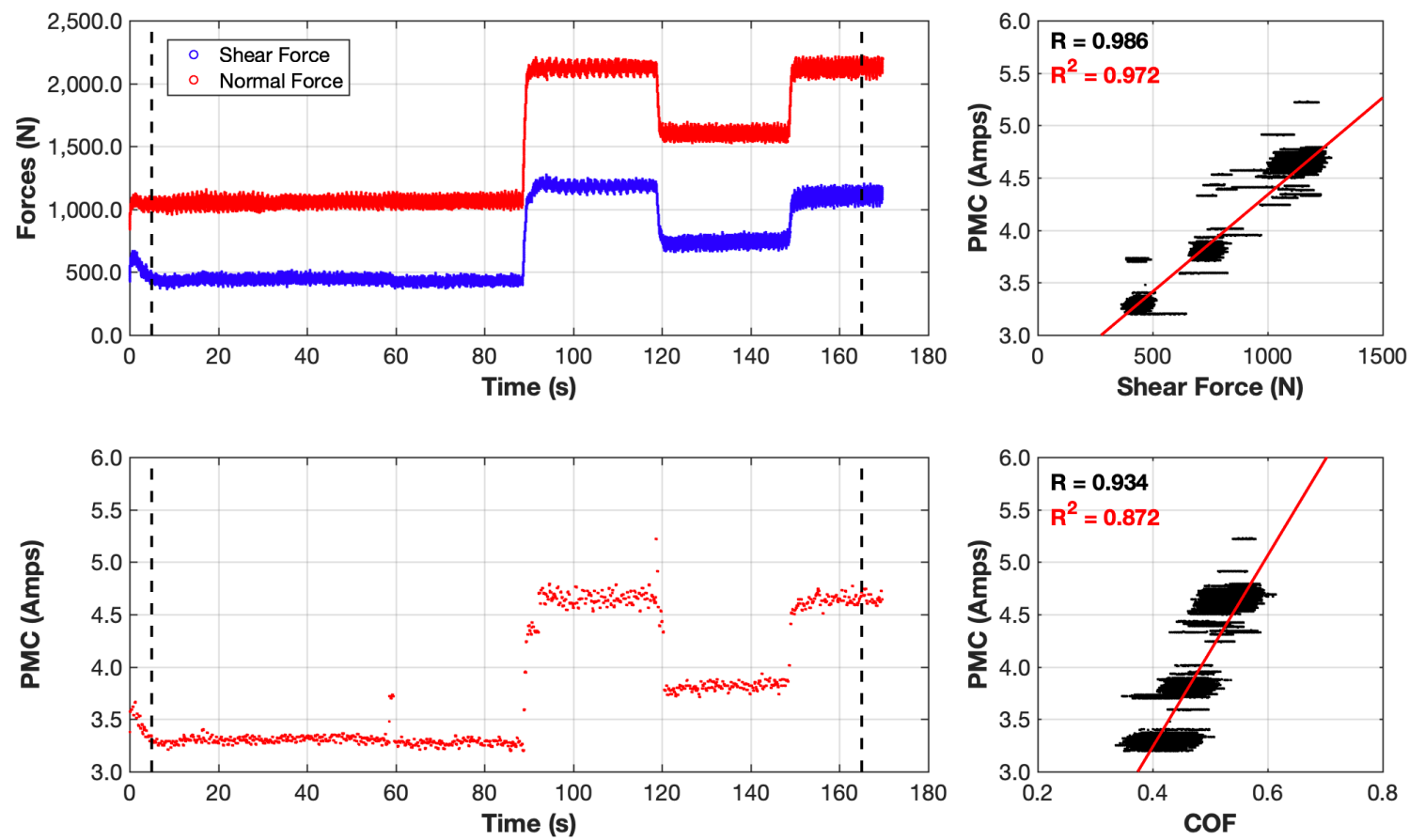

Figure 3. Case 3 - Shear Force and Normal Force vs. Time (top left), Shifted PMC vs. Time (bottom left), Correlation Plot of Shifted PMC Data vs. Shear Force Data (top right), Correlation Plot of Shifted PMC Data vs. COF Data (bottom right).
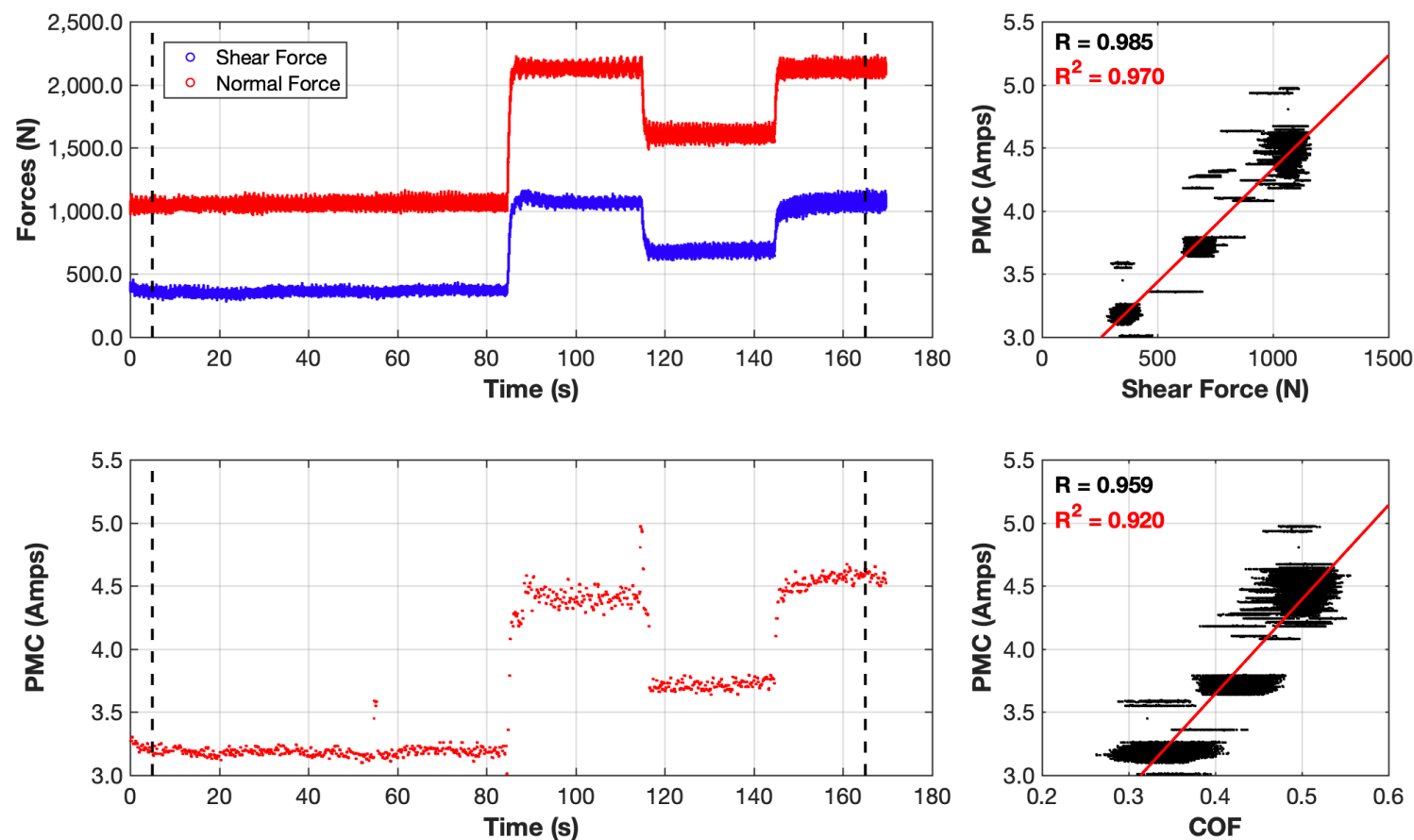

Figure 4. Case 4 - Shear Force and Normal Force vs. Time (top left), Shifted PMC vs. Time (bottom left), Correlation Plot of Shifted PMC Data vs. Shear Force Data (top right), Correlation Plot of Shifted PMC Data vs. COF Data (bottom right). 

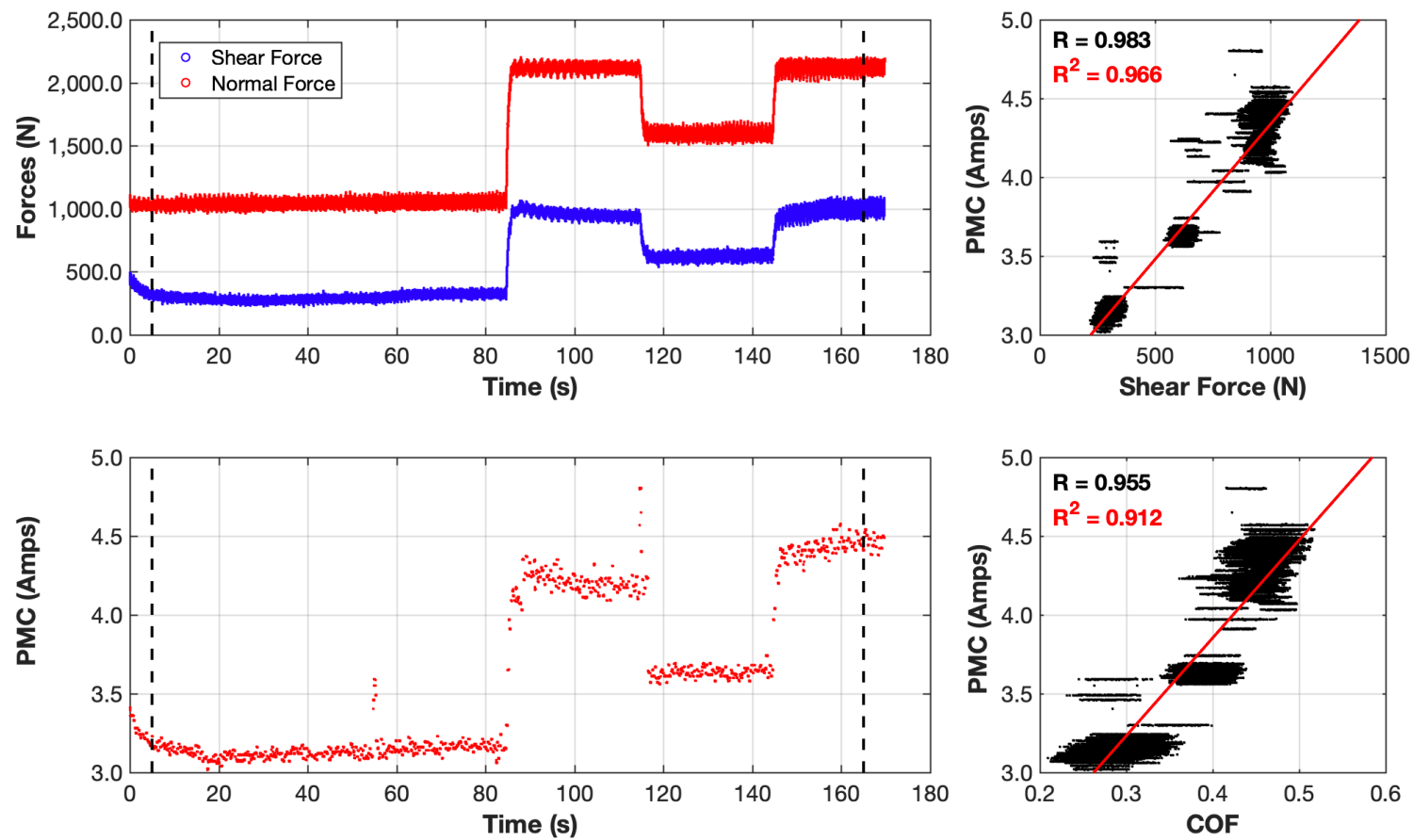

Figure 5. Case 5 - Shear Force and Normal Force vs. Time (top left), Shifted PMC vs. Time (bottom left), Correlation Plot of Shifted PMC Data vs. Shear Force Data (top right), Correlation Plot of Shifted PMC Data vs. COF Data (bottom right).
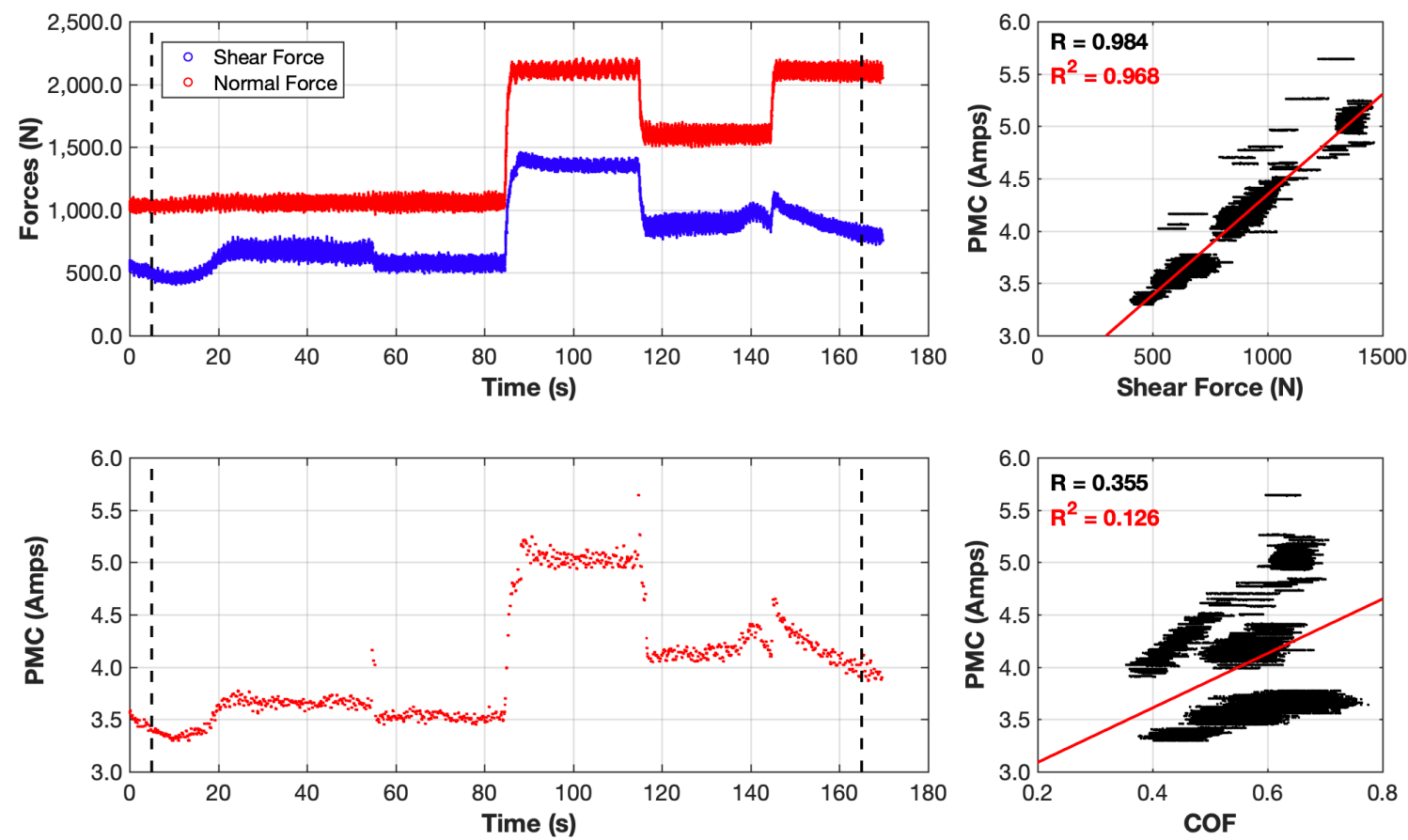

Figure 6. Case 6 - Shear Force and Normal Force vs. Time (top left), Shifted PMC vs. Time (bottom left), Correlation Plot of Shifted PMC Data vs. Shear Force Data (top right), Correlation Plot of Shifted PMC Data vs. COF Data (bottom right). 

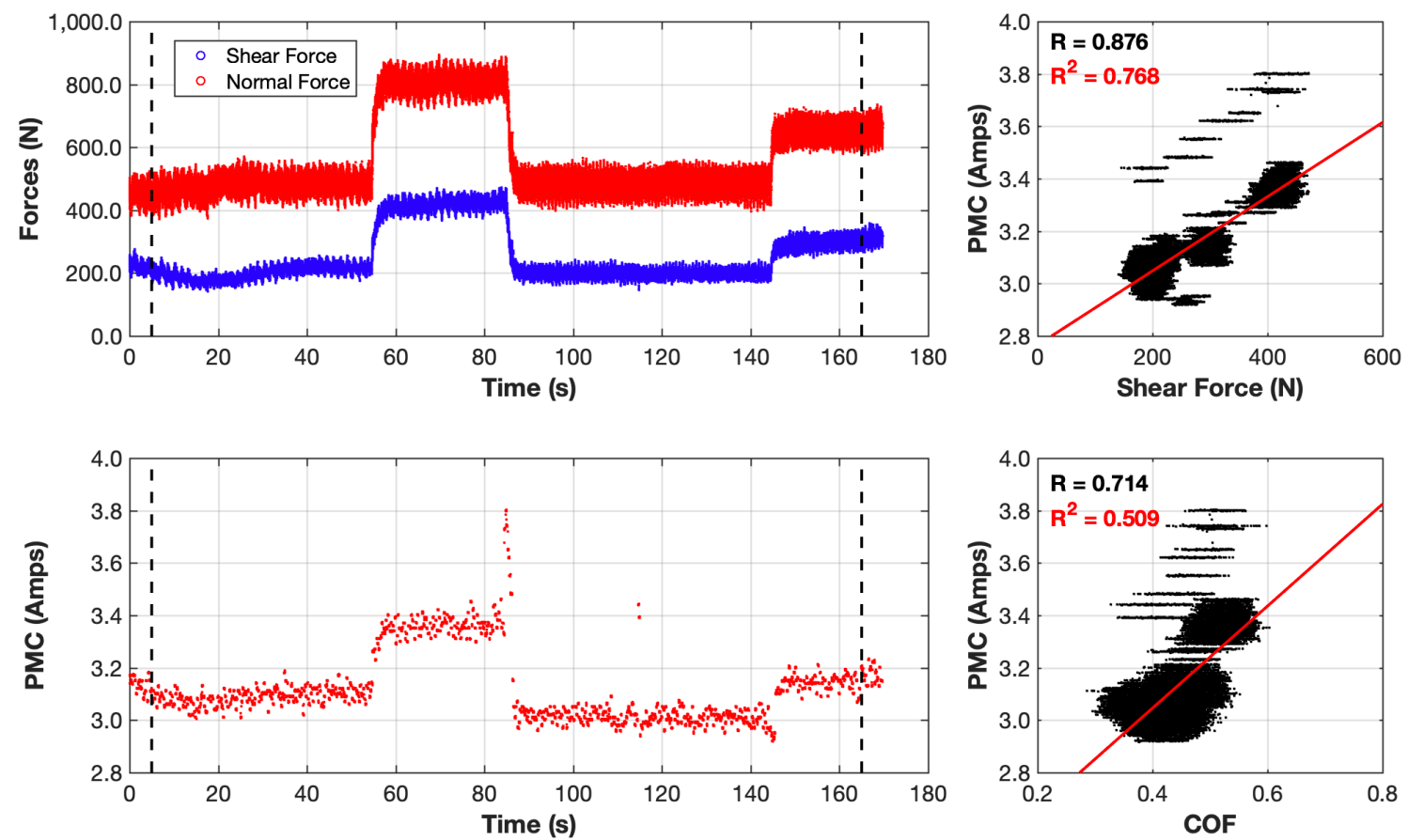

Figure 7. Case 7 - Shear Force and Normal Force vs. Time (top left), Shifted PMC vs. Time (bottom left), Correlation Plot of Shifted PMC Data vs. Shear Force Data (top right), Correlation Plot of Shifted PMC Data vs. COF Data (bottom right).
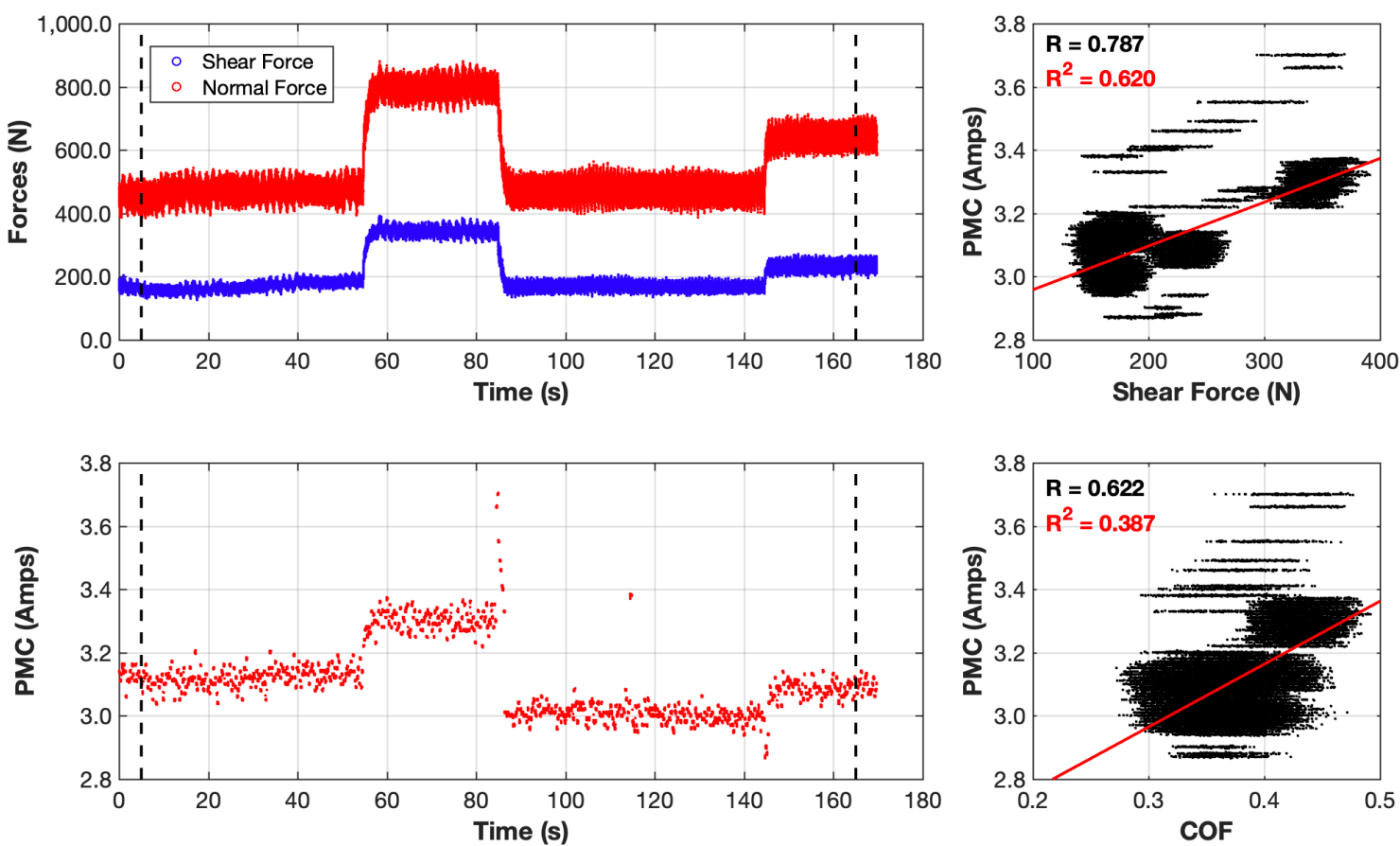

Figure 8. Case 8 - Shear Force and Normal Force vs. Time (top left), Shifted PMC vs. Time (bottom left), Correlation Plot of Shifted PMC Data vs. Shear Force Data (top right), Correlation Plot of Shifted PMC Data vs. COF Data (bottom right). 

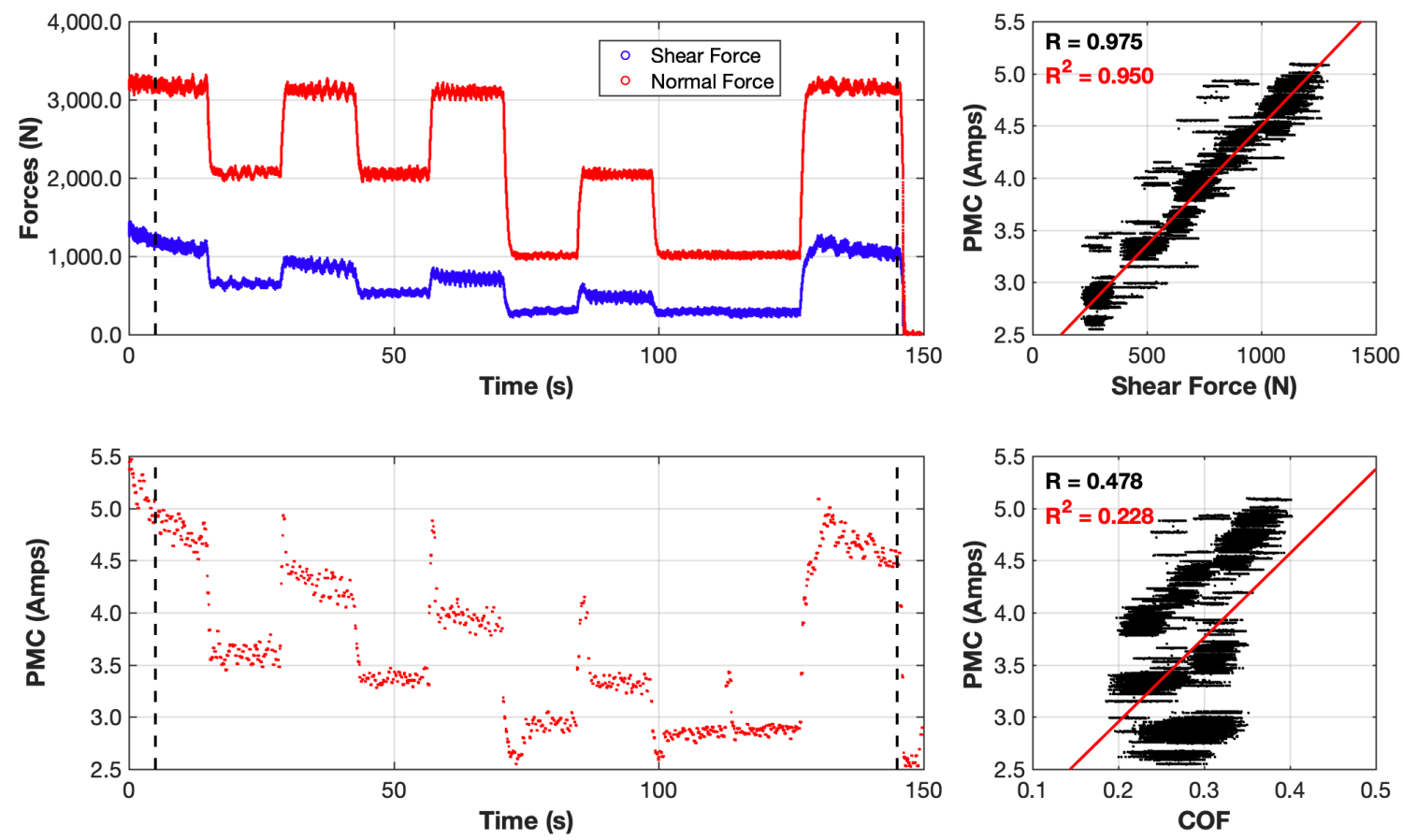

Figure 9. Case 9 - Shear Force and Normal Force vs. Time (top left), Shifted PMC vs. Time (bottom left), Correlation Plot of Shifted PMC Data vs. Shear Force Data (top right), Correlation Plot of Shifted PMC Data vs. COF Data (bottom right).
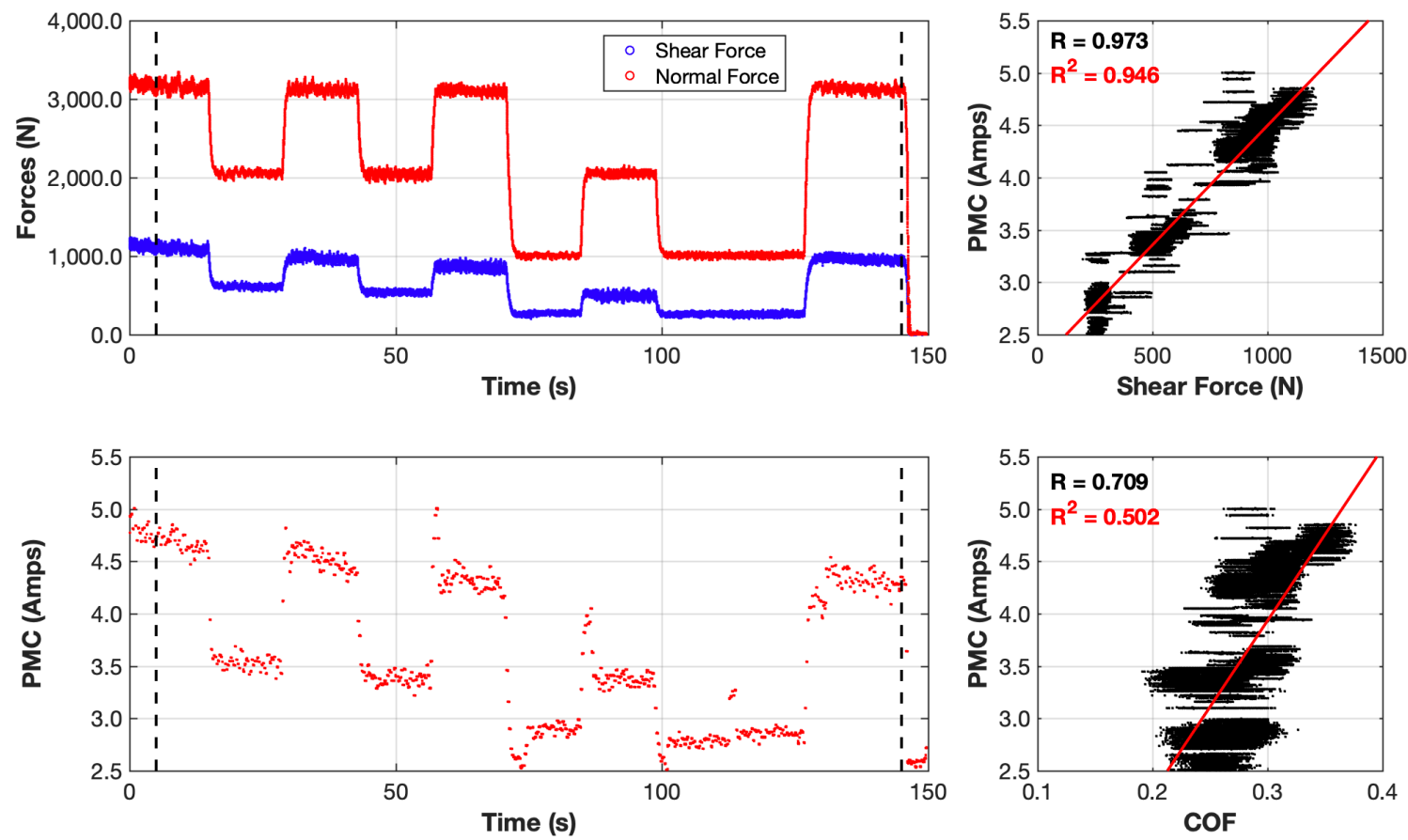

Figure 10. Case 10 - Shear Force and Normal Force vs. Time (top left), Shifted PMC vs. Time (bottom left), Correlation Plot of Shifted PMC Data vs. Shear Force Data (top right), Correlation Plot of Shifted PMC Data vs. COF Data (bottom right). 

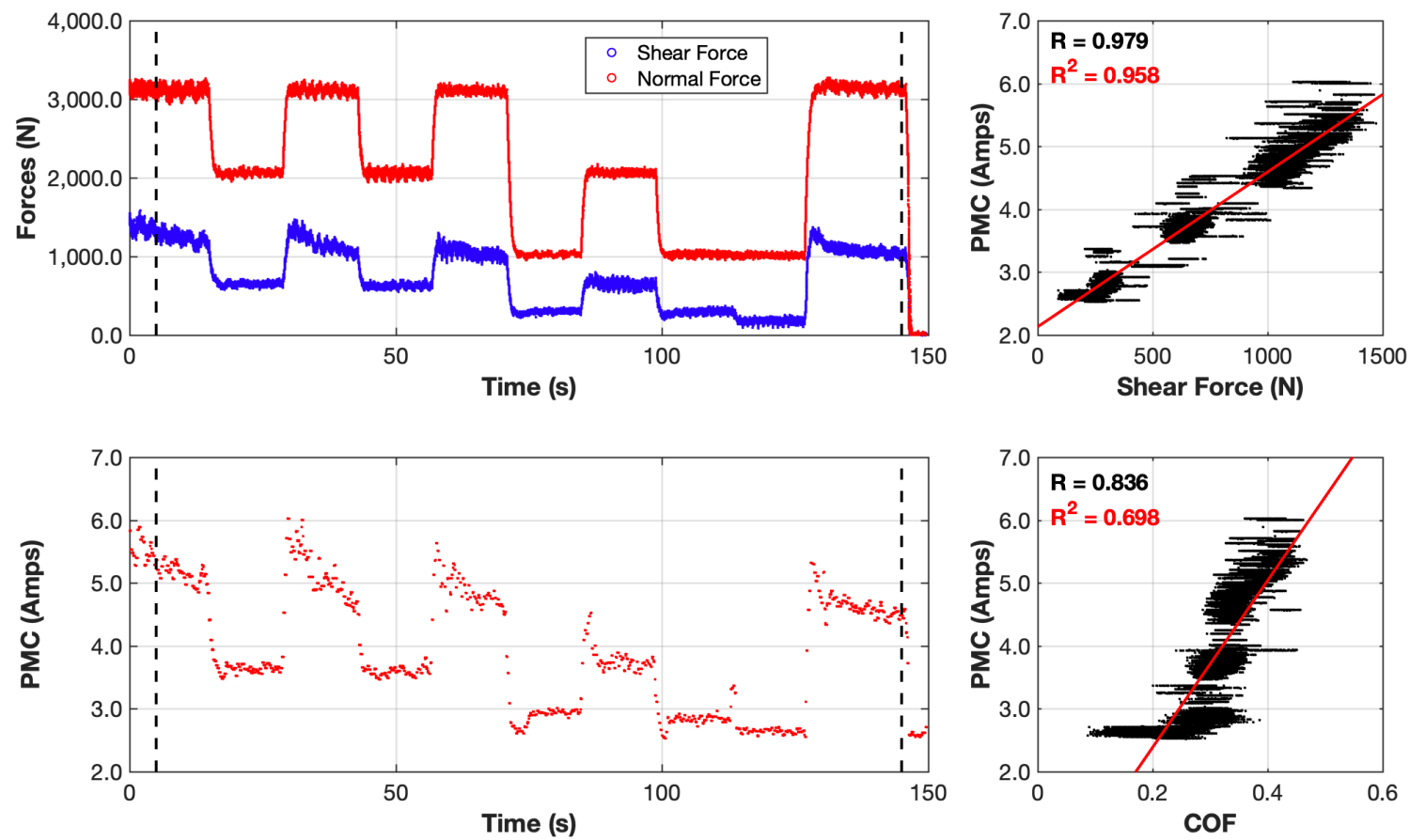

Figure 11. Case 11 - Shear Force and Normal Force vs. Time (top left), Shifted PMC vs. Time (bottom left), Correlation Plot of Shifted PMC Data vs. Shear Force Data (top right), Correlation Plot of Shifted PMC Data vs. COF Data (bottom right).
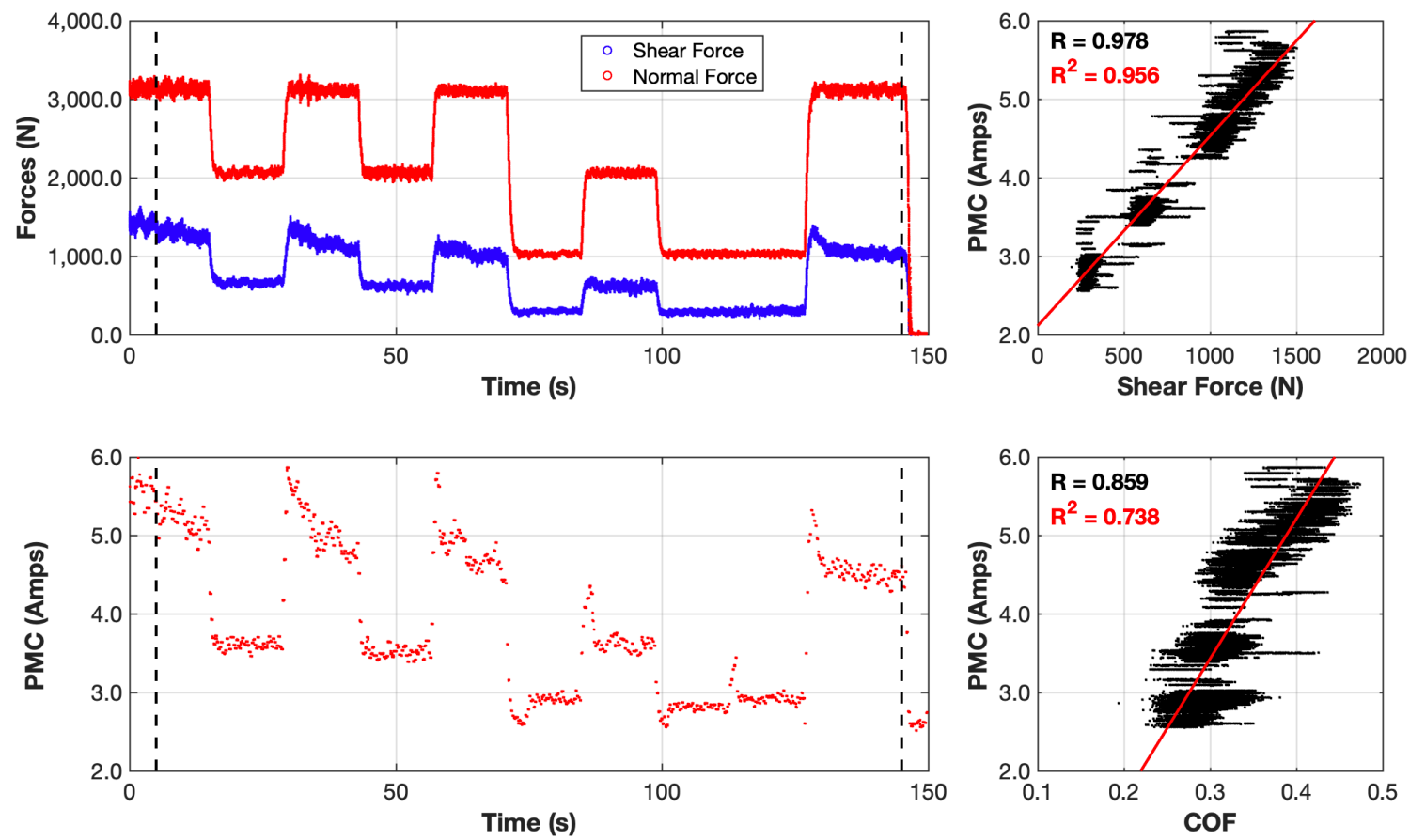

Figure 12. Case 12 - Shear Force and Normal Force vs. Time (top left), Shifted PMC vs. Time (bottom left), Correlation Plot of Shifted PMC Data vs. Shear Force Data (top right), Correlation Plot of Shifted PMC Data vs. COF Data (bottom right). 

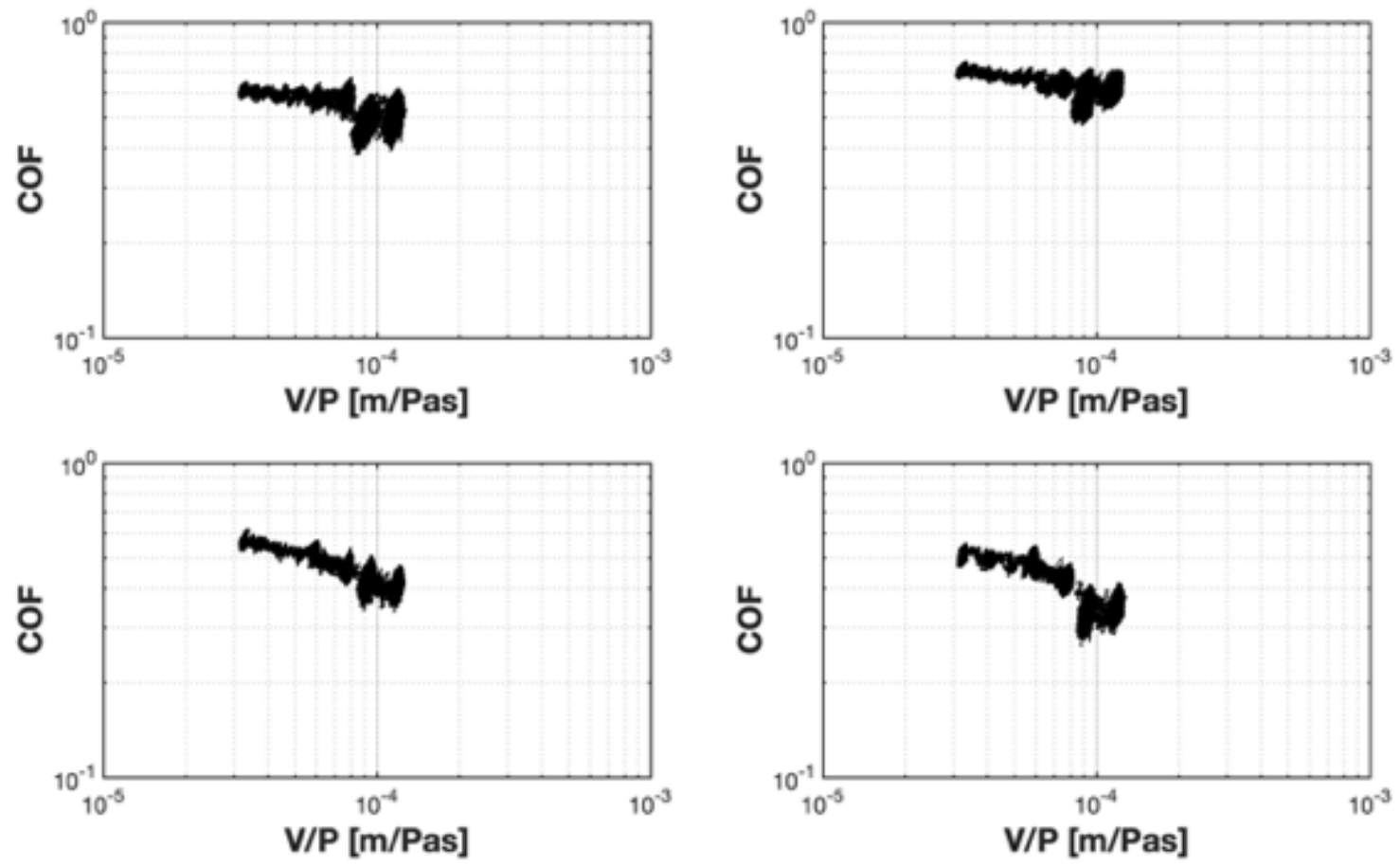

Figure 13. Stribeck+ curves for. (clockwise from top left) Cases 1-4.
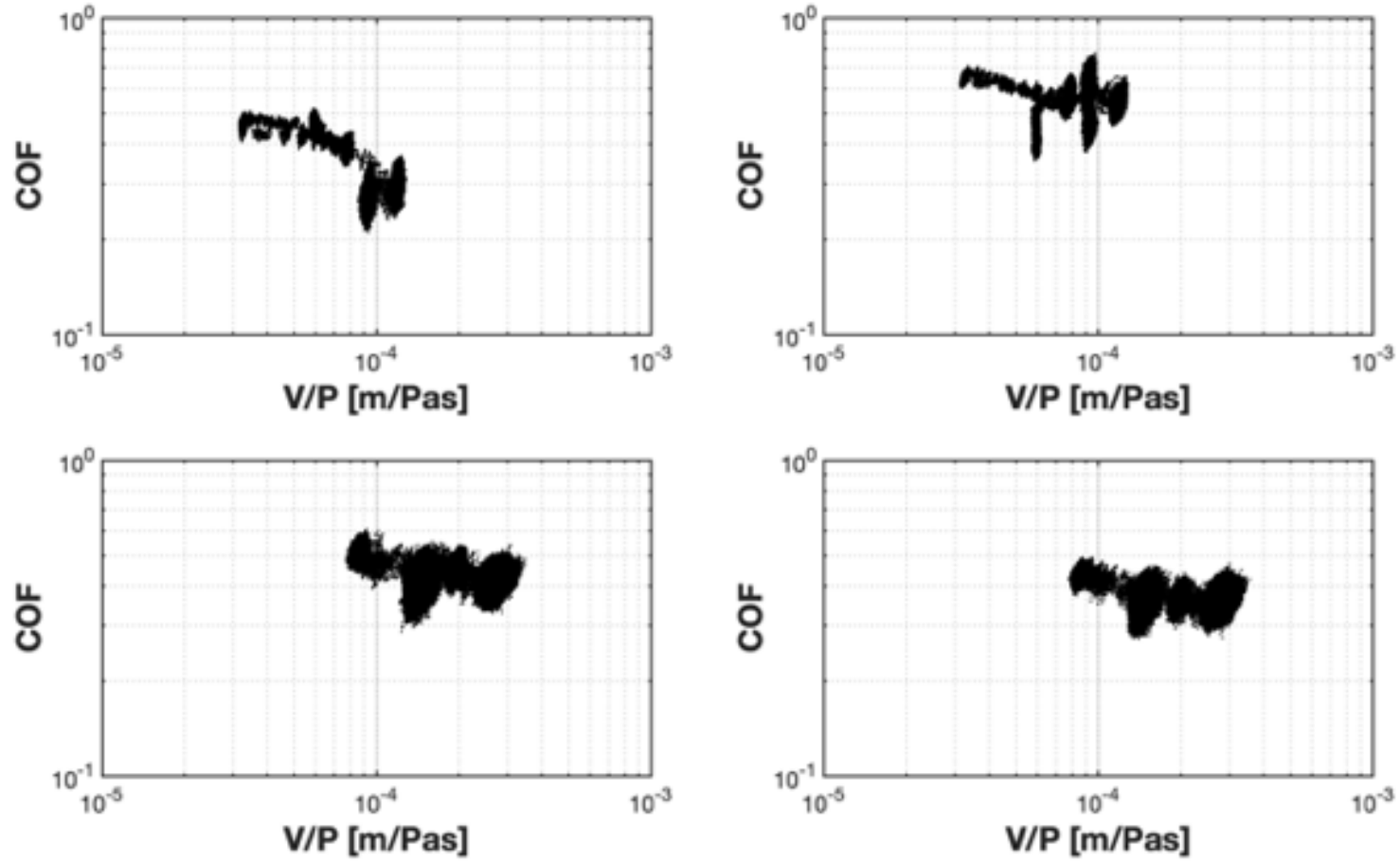

Figure 14. Stribeck+ curves for. (clockwise from top left) Cases $5-8$. 

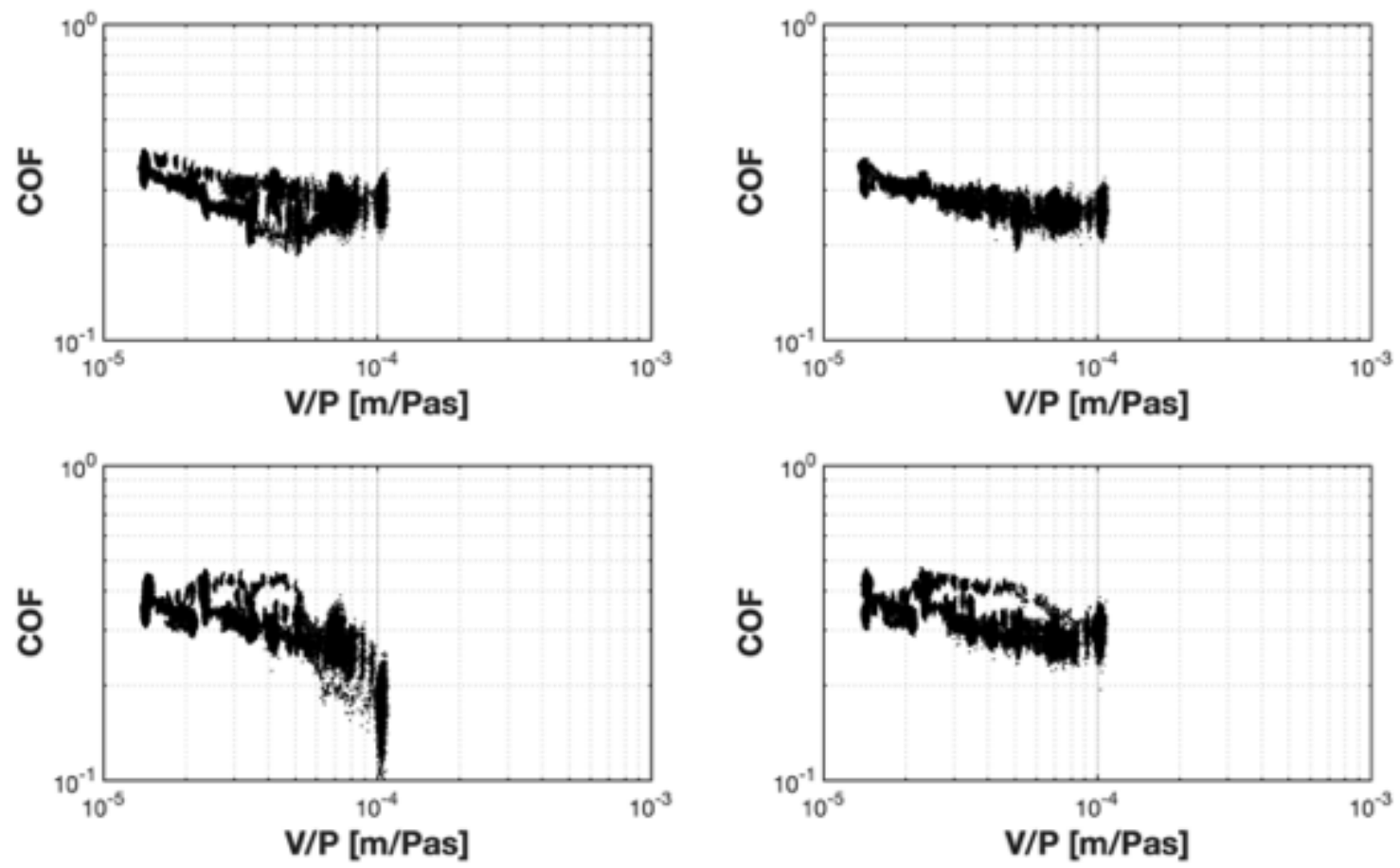

Figure 15. Stribeck+ curves for. (clockwise from top left) Cases $9-12$. 